\title{
Machine Learning Methods for Analysing the Impact of Social Media on Students Academic Performance
}

\section{Kiran Shahzadi' ${ }^{1}$, Muhammad Shahzad Sarfraz' ${ }^{1}$ Mazhra Saqib ${ }^{2, *}$, Hamza Mansoor Shah $^{3}$}

${ }^{1}$ Department of Computer Science, Faculty of Computing and Information Technology, University of Gujrat, Gujrat, 50700, Pakistan.

${ }^{2}$ Department of Computer Science and Information Technology, University of Lahore, Lahore, 54000, Pakistan ${ }^{3}$ Department of Computer Science and Information Technology, University of Lahore, Islamabad Campus, 44000, Pakistan

\begin{tabular}{llllll}
\hline Received: 02.12 .2020 & $\bullet$ & Accepted: 20.12.2020 & $\bullet$ & Published: 30.12.2020 & • Final Version: 30.12 .2020 \\
\hline
\end{tabular}

\begin{abstract}
Today Social networking is a common approach, mostly along with youngster. The social media impact on the academic performance of students become fundamental part to determine. Social Networking Sites (SNS) for example, twitter facebook are presently an vital medium that are used to connect peoples and different associations approximately the world Technology is thriving quick with the passage of time, and the youngsters are trapped in this swift revolutionize. In this paper we find out in which ways social sites affects student's academic performance and recognize the impact of social sites on our educational system. The reason at the back huge use of social networking sites. Social sites networking (SNS) have grow from being merely libertine stages for personal use to awesome progressive structure that utilized both blend and inside and remotely for relationship and joined exertion with shafts separated stakeholders. Generally among youthful understudies, Social sites networks is an unmistakable example today In this paper by using studentpor.csv and student-mat.csv dataset, we work on the classifier on the social media impact on students academic performances. For this purposes we choose K nearest-neighbor (KNN), support vector machine (SVM) and Linear regression algorithm using python that predict slightly better and give right prediction about the student's academic performance. In social networking model our work has enhanced our imminent. The results reveal that there is strong relationship between social media and student's academic performance. This paper shows that mostly students have cell phones and also have internet availability so they use 40 mints form 4 hours daily and not give attentions to their studies, all these activities on internet effect the student's academic performance. This study will commence with introduction and background of existing studies. After that on the basis of background there will be a research question.
\end{abstract}

Keywords: Social Websites, Face book, Social Media, Study, result, Social Networking Sites

\section{Introduction}

From all around the world social frameworks have transformed into an around the globe contemplate in wide individuals in a variety of ages, learning levels, human advancements thus forward [1]. The characterization of social media is "shows the relationships between groups of peoples". In the past some years, significantly online community has been changed. It's a grateful discovery of social media, people of all the ages' men and women nowadays switch over or communicate their plans,

* Corresponding Author: ahmad15001820@gmail.com 
thoughts, personal information, snaps and videos surprisingly. On the other hand, every day, huge number of students is spending immeasurable time and deeply involved with social media, like Face book, Twitter MySpace and other social sites . At first give the impression of being this may seem like totally wastage of time. Conversely it also provides facilities to students to develop their knowledge and social skills, and be like a vigorous civilian who create and share content. Presently, even if social media is appropriate or adverse. On the daily basis huge numbers of students visit these sites. As social media sites become popular day by day with unbelievable speed it is our faith for student achievement equation technology is a fundamental part. As students in Twitter, Facebook, and other social sites share gossips from newspapers, updates about TV or magazines and also announce novelty advertisement, new brands or occurrence notifications connected to musical concerts, movies, dramas, entertainment and games activities etc. an additional most important usage factor revealed out study as 'content' and 'status update' factor. Because of the intrinsic mobile nature of these devices, people of various ages are able to converse and connected with one another and access social networking sites at any moment and in any place [2]. On the other hand, this ease and rate of recurrence of use may have poisonous penalty for society on the whole. Students now socialize through using internet and it takes absent from the person socialization that has been around forever.

Affected social networking websites Just as we communicate by changing the way we interact face to face, how we get information and dynamic of our social groups and friends. Many ancestor are anxious that their child's are wasting too much time on Twitter and other social networking sites and not give enough time to their studies. For that reason, in this paper we determine the social media impact on academic performance of students, and find out in which ways social sites affects student's academic performance and recognize the impact of social sites on our educational system. Despite regularly reviewing the messages on different social sites, analyzing bit by bit or get together, in addition to reviewing the newspapers daily, now people also check their profiles on social networks by changing the rank of others, publishing their summary [3].

For more reasons, number of people use the SNS “Allows to quickly update, search and share without any restrictions without maintaining facilities, daily life, builds the social contacts and connection, With the help of collaboration and posts, the learning of maintaining comfortable learning conveyance and encouraging instruction is important". Leading writers describe that social networks are using different people to about wastage of time, people and their culture. In addition, in the case of office work or social work, the social network plays an important role. Meet new people who know their interests or political matters said by Ellison [4].

The immense majority of social networking sites are a large part of the today's young generation, which are university students. Consequently, it is assumed that SNS plays an active role in daily life of the young generation.[5]. Similarly, comparing different SNS with respect to its prominent aspects and the use of social economics, for example, gender, the recurrence of the use and its purposes naming are interested in the environments of social networks remain as the most common research region[6,11].

This paper sorts out as Area 2 will cover the problem statement. Segment 3 will cover Dataset Characterization. In Area 4 we will describe Tool Description. Area 5 covers the methodology, 6 portions define limitations and future work and in the last we define conclusion. 


\section{Problem Statement}

The fast improvement of media innovation has hugely influenced the mode network speak once ordinary. Social networks are one-way People feel they are related to the community. Due to its popularity economist and teachers raised the question is whether student's academic performance will be affected if they spend time on those sites. Choney [7], Mehmood et al [8] Be sure to use technology like internet is one of the most important factors that can affect educational performance Students positively or negatively. To address the problem of using social networking, first of all the question raised in this study is: What is the purpose of the student's social media use? Investigations on this topic will begin to show that social networking sites are easily available Part of this talk to students without interacting on grades. Such, the purpose of this research is to discover the benefits and damages of the student's social media utilization[9].It's neighboring the base establishment that this research is deciding the aftereffect of the utilization of student's social networks on their educational activities. Study performance of students Can affects by social network?

\section{Data Characterization}

The data were obtained in a survey of students math and portuguese language courses in secondary school. It contains a lot of interesting social, gender and study information about students. There are two files of data set, student-por.csv and student-mat.csv , $1^{\text {st }}$ file contain 650 instance and $2^{\text {nd }}$ file contain 396 round about total instances are 1 Thousand and forty four(1,044). Data set is multivariated. Number of attributes are 33. Attribute Characteristics are Integer/Categorical. Dataset was created in April, 2008, we extract the data in May,2018.

\section{Tool Description}

Python is a standout amongst the most protractile and well-known tool used in machine learning field for classification. In this algorithm, collection of source and in addition pre-processing, grouping and information extraction is done by using "Python" tool.

\section{Methodology}

\subsection{Support Vector Machines (SVM)}

bolster vector machines (SVM) is an administered learning model, that is related with learning algorithm that dissect information utilized for relapse and classification.(SVM) isolates the information directly. It was presented in 1992.It is known as twofold classifier and it can likewise use for multiclass arrangement. utilizing hyper design it isolate the classes, that is most detachable line used to part the classes information, (Figure 1) like other directed grouping calculations SVM utilizes class marked information in preparing stage. SVM maps the information into highlight space and by the hyper design split the information into its classes that has most prominent edge among the occurrences of the classes. it is effective in high dimensional spaces and where number of measurements is more noteworthy than the quantity of tests. Two various techniques, one-versus one and one-versus all are utilized for multiclass order. SVM use hyper plane for isolated information directly but not every one of the information are straightly divisible, appeared in (Figure 2).To contract with that kind of information (SVM) maps the information highlights into high measurement space utilizes bit trap, to make the information detachable linearly. Four fundamental portion capacities are utilized Direct, Polynomial, Outspread and sigmoid. 


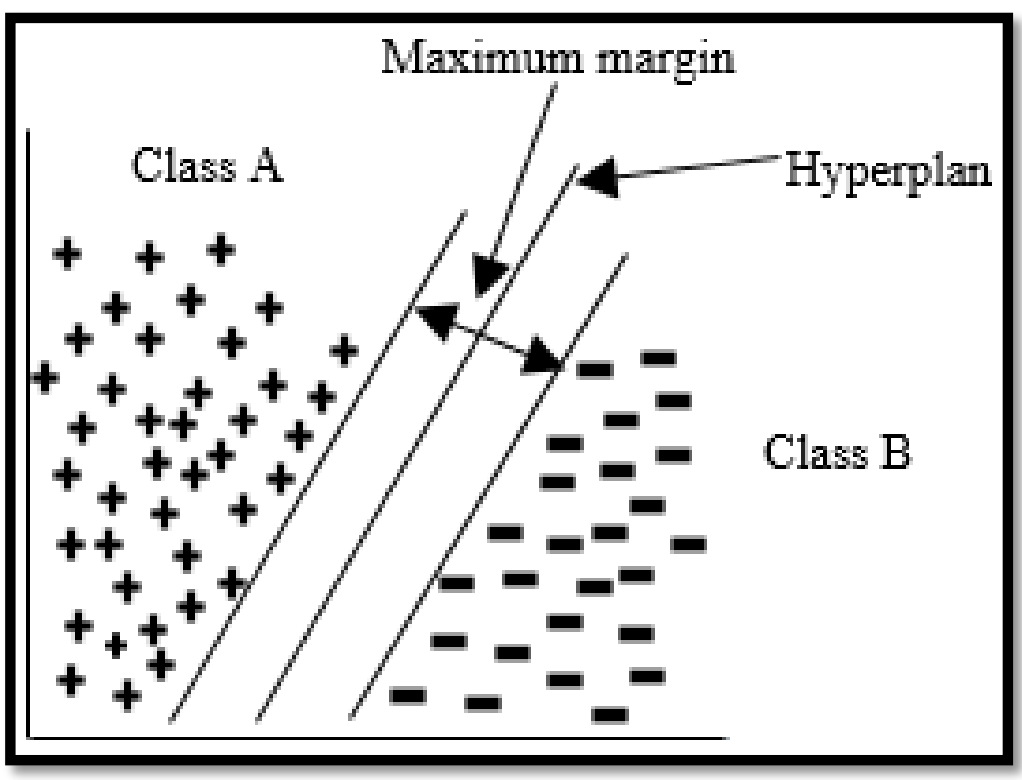

Figure 1. Linearly separable data [10].

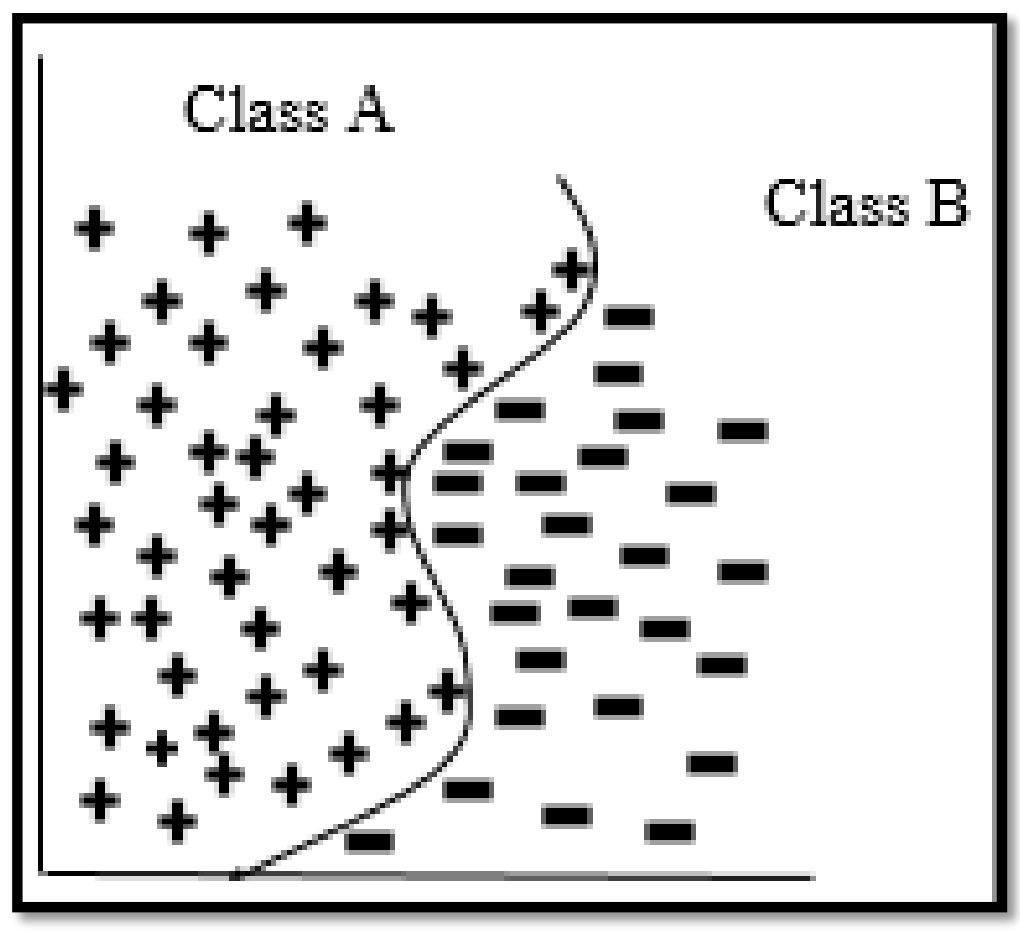

Figure 2. Linearly non-separable data [10].

\subsection{K-Nearest Neighbors (KNN)}

KNN (K-Nearest Neighbors algorithm), KNN algorithm is one of the most used learning algorithms and one of the simplest classification algorithm, Yet with that simplicity, it can give highly accurate results, it is a non-parametric algorithm to classify samples. $\mathrm{KNN}$ is useful for both regression or classification but mostly it used for classification problems.for represtation KNN stores entire training dataset.KNN is also known as instance based learning. on the input vectors It calculate the fairly accurate distances between different points, does not contain the model, only searches the examples and classifies new instances. Thus, k-NN "on-line" trains the examples and finds knearest neighbor of the new instance. and then assigns the unlabeled point to the class of its KNN. 
$(\mathrm{K})$ is an important parameter and diverse $(\mathrm{K})$ values will cause diverse performances. If $(\mathrm{k})$ is significantly large, the neighbors which used for prediction will influence the accuracy of prediction and make huge classification time of prediction. IN KNN training stage.

\subsection{Linear Regression}

Where the number of independent variables is one and there is a linear relationship between the dependent (y) and independent (x) variable linear regression. It is a type of regression analysis, it concerns two-dimensional sample dependent and independent. and finds a linear function that, as accurately as possible. the slope of the fitted line is equal to the correlation between $y$ and $x$ corrected by the ratio of standard deviations of these variables It is extremely straightforward yet useful algorithms in machine learning.

\section{Results and Discussions}

We have two dataset files which have 1 thousand forty four approximately data in each of the dataset files. According to our hypothesis we are required to firstly the social media effect on student and then we have to classify performance of a student in study. We choose dataset files for classification of social media effect on students. We apply three different classifiers e.g. support vector machine(SVM), K nearest neighbor(KNN) and Linear Regression . For detecting the effect of social media , we use dataset file $1^{\text {st }}$ for the training set and get their model and use $2^{\text {nd }}$ dataset file for testing and save the results after testing Result of social media effects by using Dataset as testing (Figure 3-11) and (Table 1).

Table 1. Standard Deviation Results

\begin{tabular}{|l|l|l|l|l|l|l|}
\hline \#sr & age & Medu & Fedu & trveltim & studytim & faliure \\
\hline count & 395.0000 & 395.000 & 395.0000 & 395.0000 & 395.0000 & 395.000 \\
\hline mean & 16.69620 & 2.74936 & 2.521519 & 1.448101 & 2.035443 & 0.33417 \\
\hline std & 1.276043 & 1.09473 & 1.088201 & 0.697505 & 0.839240 & 0.74365 \\
\hline min & 15.00000 & 0.00000 & 0.000000 & 1.000000 & 1.000000 & 0.00000 \\
\hline $\mathbf{2 5 \%}$ & 16.00000 & 2.00000 & 2.000000 & 1.000000 & 1.000000 & 0.00000 \\
\hline $\mathbf{5 0 \%}$ & 17.00000 & 3.00000 & 2.000000 & 1.000000 & 2.000000 & 0.00000 \\
\hline $\mathbf{7 5 \%}$ & 18.00000 & 4.00000 & 3.000000 & 2.000000 & 2.000000 & 0.00000 \\
\hline max & 22.00000 & 4.00000 & 4.000000 & 4.000000 & 4.000000 & 3.00000 \\
\hline
\end{tabular}




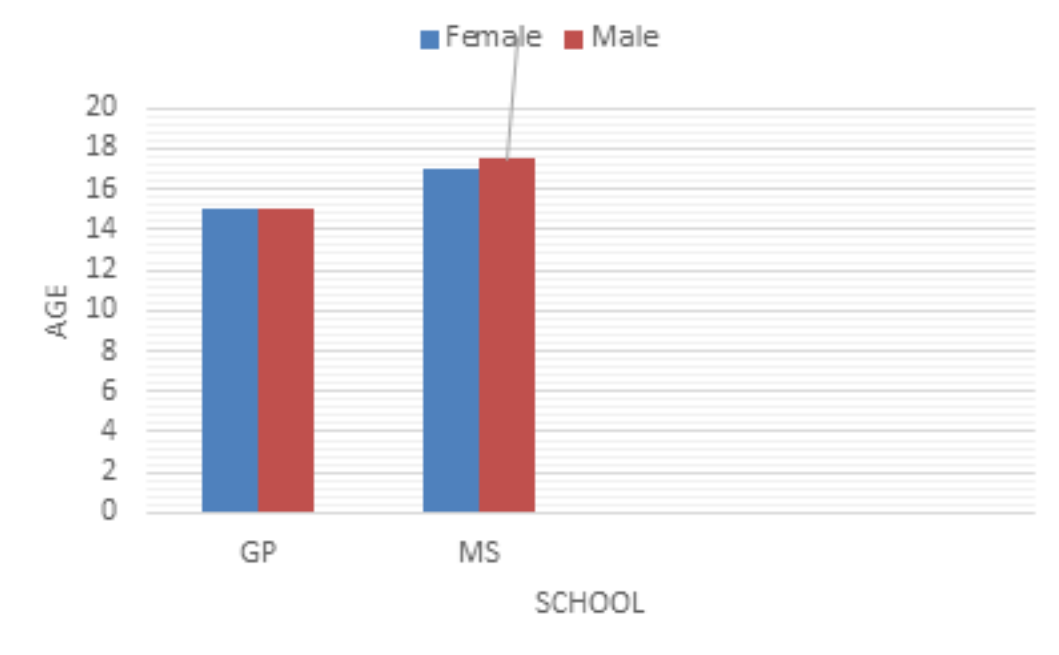

Figure 3. Results depicting the relationship between school and age.

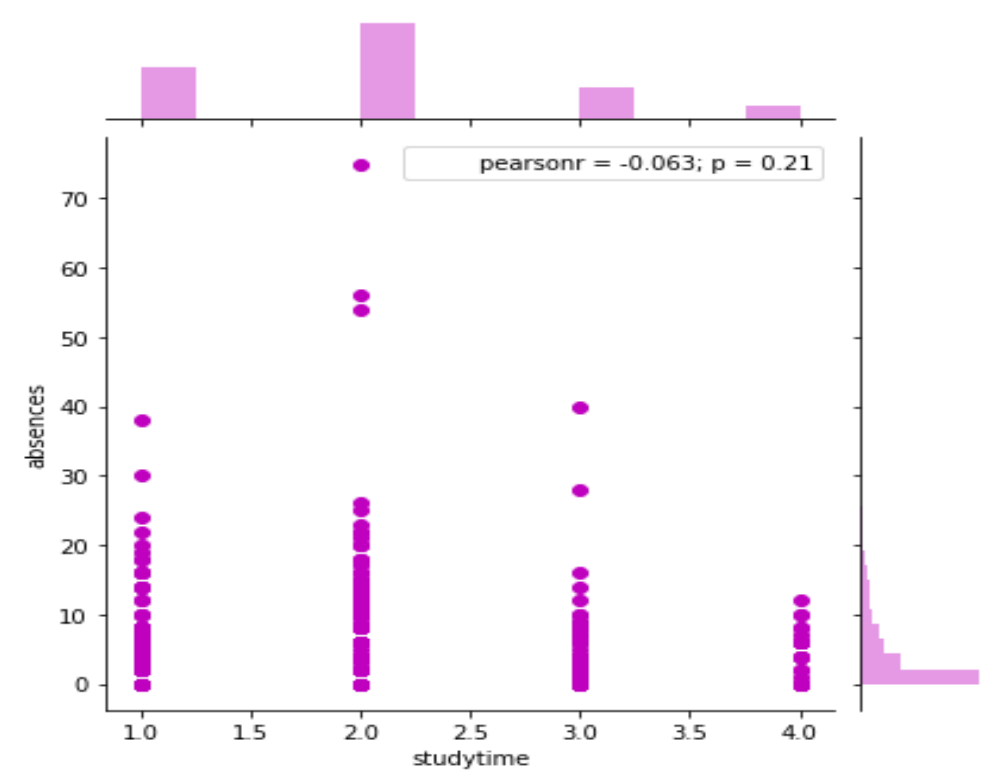

Figure 4. Result OD Absence and Studytim.

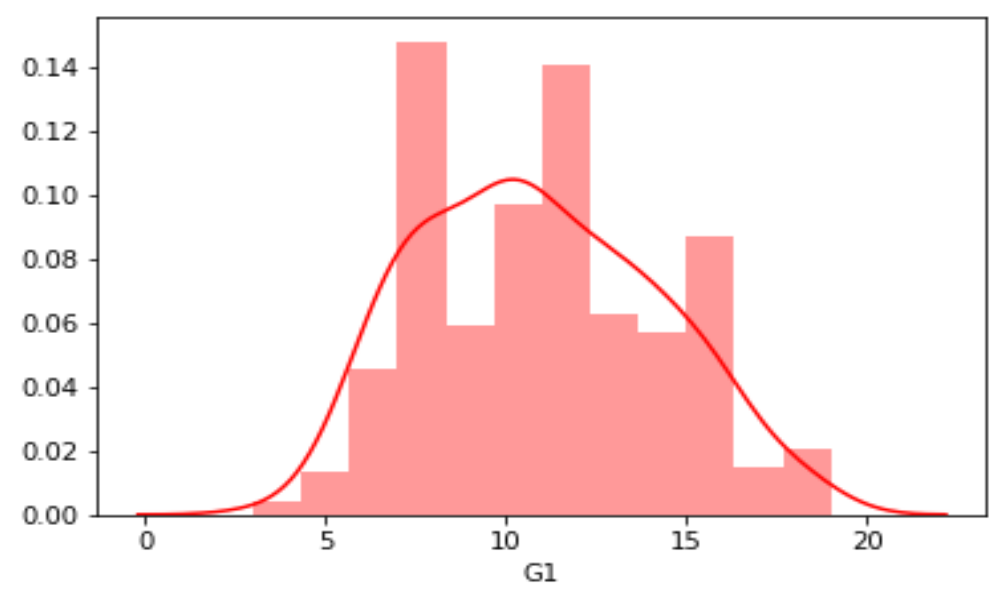

Figure 5. G1 results. 


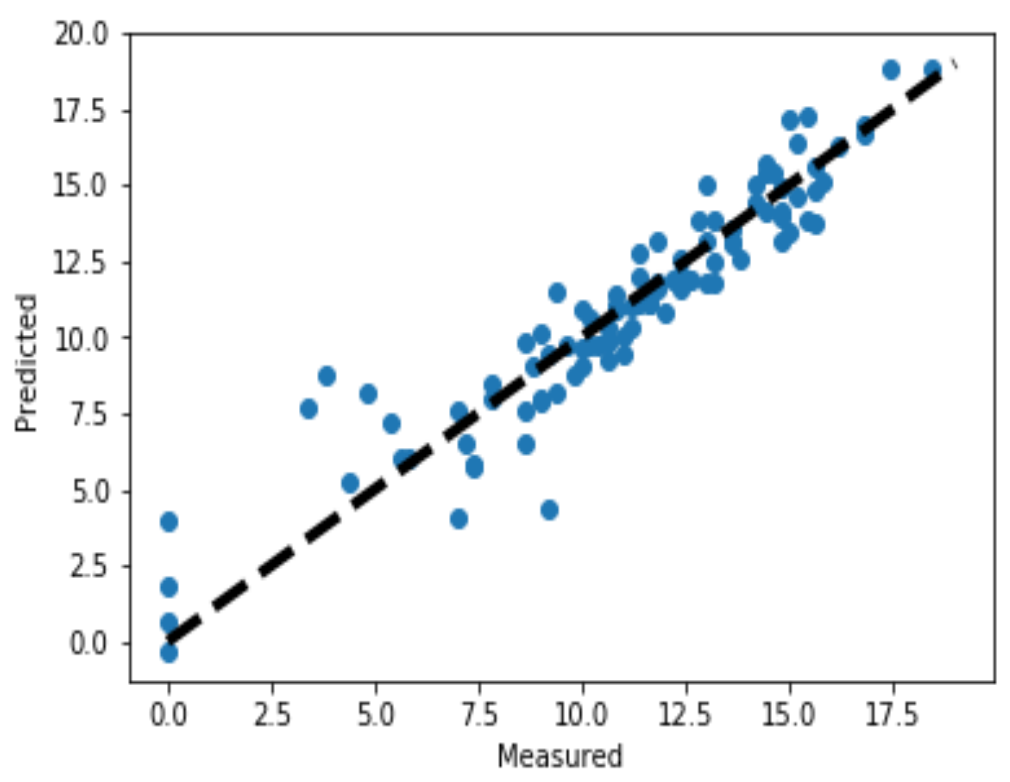

Figure 6. Relationship between predicted and measured.

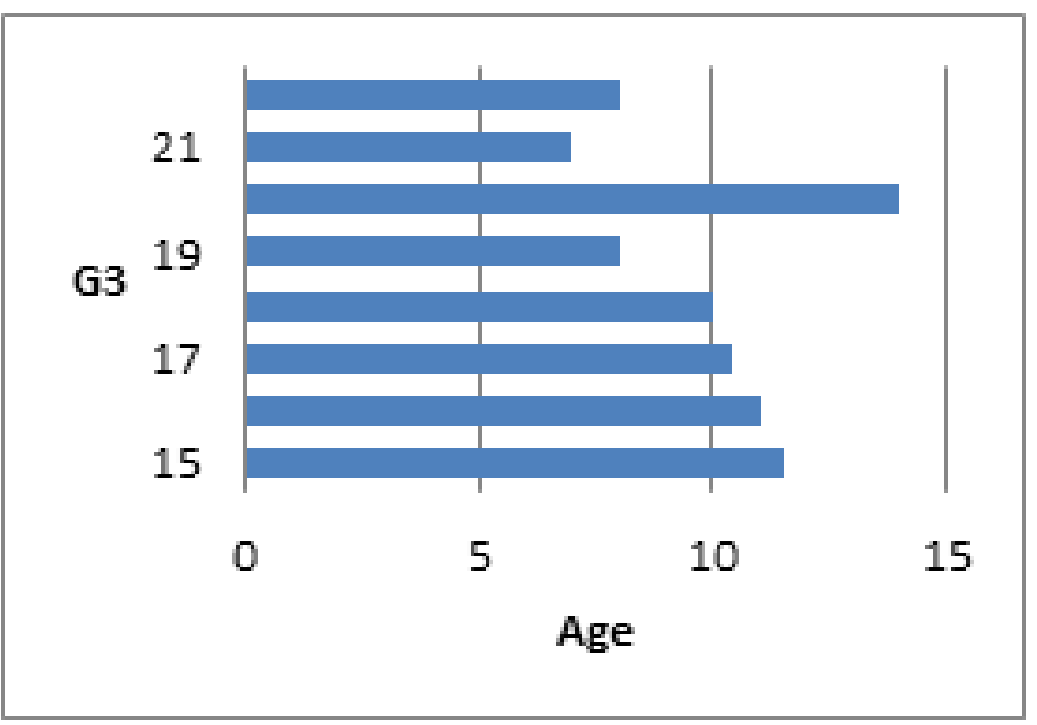

Figure 7. Relationship between G3 and age. 


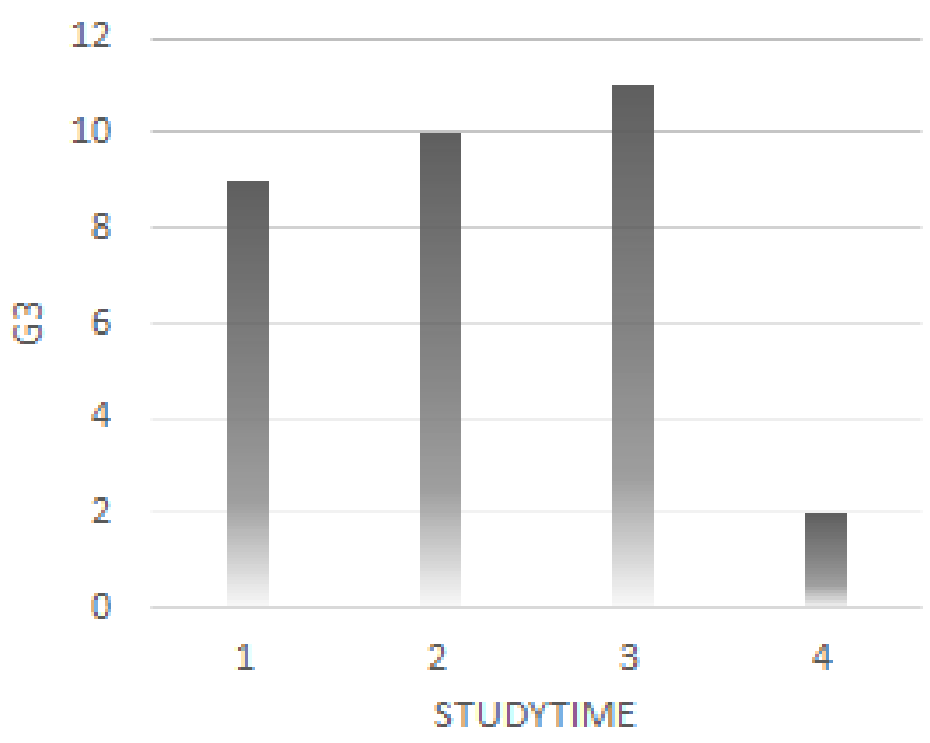

Figure 8. Relationship between G3 and study.

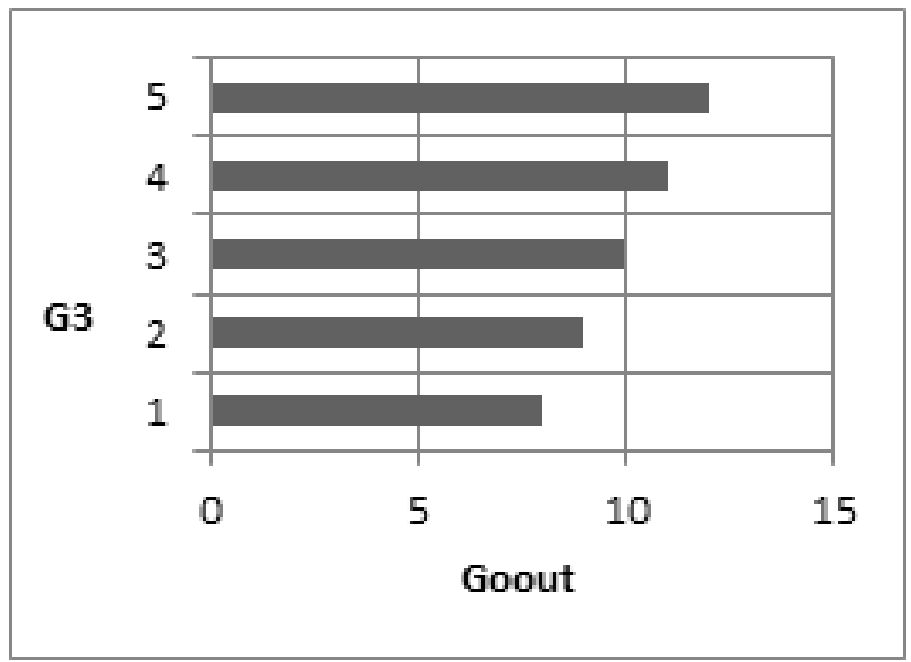

Figure 9. Relationship between G3 and Goout.

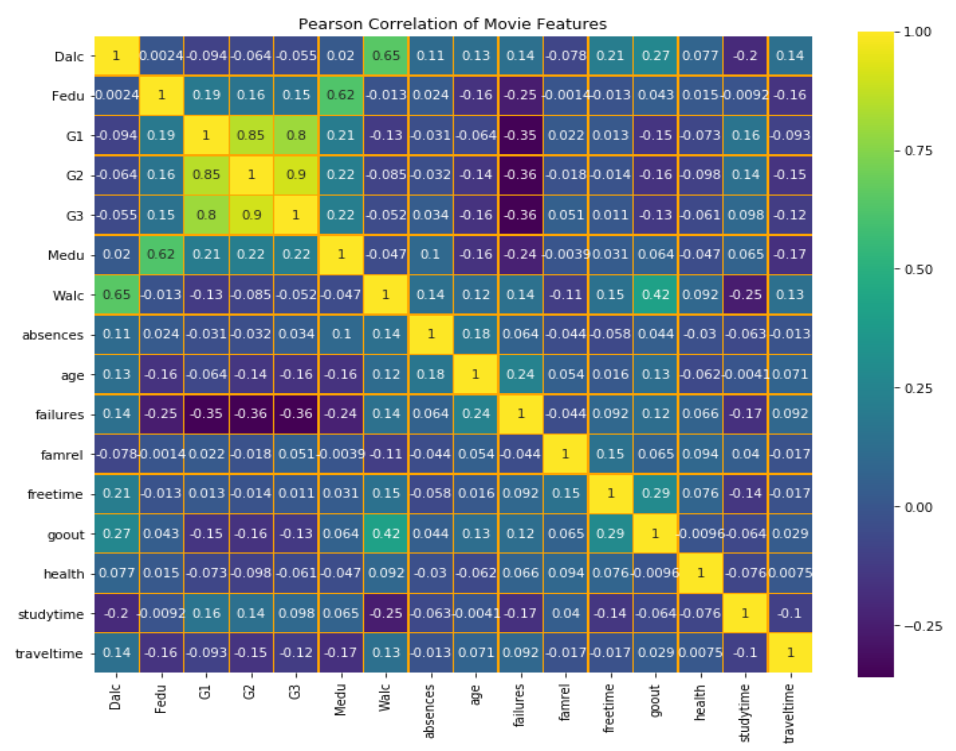

Figure 10. Features Correlation. 


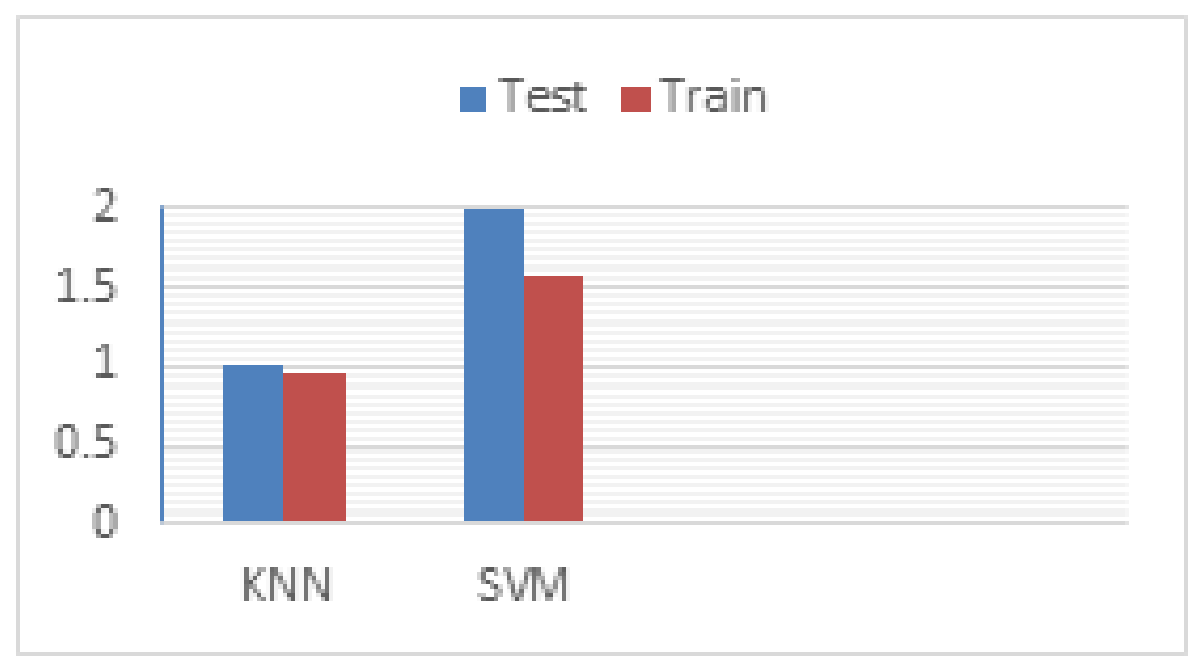

Figure 11. Accuracy model of KNN and SVM.

\section{Limitations and Future Work}

Limited range of respondents is the first drawback of this research that were approach to experiment the effects of social media. As an overview there is no $100 \%$ accuracy in the relationship between student academic performance and usage of social sites. Secondly still it was tricky to find out origin to build on as the majority of the student's academic performance affected by the social media impact or the effects it causes perceptively. Future work it must speak to specific goals, better use of social resources and contents they occupied in on different social sites for an exact description of the effects of the actions on academic performance on students and apparent social media grip.

\section{Conclusions}

The Analysis was accomplished to explore the affects on student's academic performance of social media. Dataset that was used in this paper to examined the social media impact on student performance in educational activites. The analysis shows that most of the students have mobiles which as well have the internet facility and they familier about many social sites. In this paper, our study has exposed that by the wide-ranging use of social media affects the study performance. Social sites are appealing, excellent and popular these sites are not only offer to release some pressure of study also give them opportunity to students to make new friends. It's a vital need to provide accessibility of academic material and other novels related to studies for the students that can helped them in to their academic success just because the use of media had unenthusiastically affected the academic effort of students.

\section{References}

[1] N. A. Jonson, "Looking at', 'Looking up' or 'Keeping up with' People? Motives and utilizations of Facebook," CHI 2008 Proceedings: 1027-1036, 2008.

[2] Correa, T., Hinsley, A. W., \& De Zuniga, H. G. (2010). Who interacts on the Web?: The intersection of users' personality and social media use. Computers in Human Behavior, 26(2), 247-253.

[3] M. Lenhart, "Adults and social network websites. Pew Internet \& American Life Project Report. Available from http://www.pewinternet.org/pdfs/PIP_Adult_social_networking_data_memo_FINAL.pdf]” May 19, 2009. 
[4] C. Lampe, N. Ellison, \& C. Steinfield, "A Face(book) in the crowd: Social searching vs. social browsing," Proceedings of the 2006 20th Anniversary Conference on Computer Supported Cooperative Work (pp. 167170). New York: ACM Press, 2006.

[5] T. Pempek, Y. Yermolayeva, \& L. Calvert, "College students' social networking experiences on Facebook.,"Journal of Applied Developmental Psychology,30, 227-238, 2009.

[6] C. McLoughlin, \& M. J. W. Lee, "Social software and participatory learning: Pedagogical choices with technology affordances in the Web 2.0 era," Paper presented at the ASCILITE, Singapore. 664-675, 2007

[7] G. Attwell, "Social Software, Personal Learning Environments and Lifelong Competence Development. Available from http://www.knownet.com/writing/weblogs/Graham_Attwell/entries/6665854266/LLLa dple.rtf/attach/LL Landple.rtf” August 2, 2008.

[8] F. Stutzman, “An evaluation of identity-sharing behavior in social network communities,” Paper presented at the iDMA a and IMS Code Conference, Oxford, Ohio, 2006.

[9] Wang, Q., Chen, W., \& Liang, Y. (2011). The effects of social media on college students.

[10] Mehmood, T., \& Rais, H. B. M. (2016, August). Machine learning algorithms in context of intrusion detection. In Computer and Information Sciences (ICCOINS), $20163 r$ International Conference on (pp. 369-373). IEEE.

[11] Muhammad Ahmed, Mahnoor Dar, Rana M. Tahir, Fatima Masood. "Impact of social media on academic: A quantitative study", 2018 International Conference on Computing, Mathematics and Engineering Technologies (iCoMET), 2018 